\title{
Enantiomerically pure pentagonal-bipyramidal metal complexes with predetermined helicity in the solid and the solution state
}

\author{
Michael Seitz, Anja Kaiser, Sabine Stempfhuber, Manfred Zabel and \\ Oliver Reiser* \\ University of Regensburg, Institute for Organic Chemistry \\ Universitätsstr. 31, D-93053 Regensburg, Germany \\ oliver.reiser@chemie.uni-regensburg.de
}

\section{Supporting Information}

Table of Contents:

page

1. Extended crystallographic data for $2 c$ and 3-5

2. X-ray crystallographic data collection and

S6 refinement details for $2 c$ and 3-5

3. NMR spectra of compounds $(R, R)-\mathbf{1}, \mathbf{2 c}$ and $\mathbf{4}$ 


\section{Extended crystallographic data for $2 c$ and 3-5}

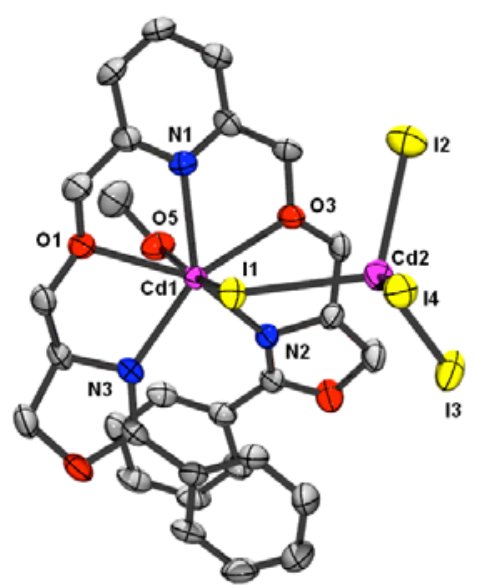

ORTEP-plot of $\left[\mathrm{Cd}(\mathbf{1})\left(\mathrm{Cdl}_{4}\right)(\mathrm{MeOH})\right](2 \mathrm{c})$ (50\% probability level, hydrogens omitted for clarity) with atom numbering scheme.

$\begin{array}{ll}\text { Cd1-N1 } & 2.395(5) \\ \text { Cd1-O1 } & 2.506(5) \\ \text { Cd1-O3 } & 2.412(4) \\ \text { Cd1-O5 } & 2.400(5) \\ \text { Cd1-N2 } & 2.396(6) \\ \text { Cd1-N3 } & 2.371(5) \\ \text { Cd1-I1 } & 2.9719(7) \\ \text { Cd2-I1 } & 2.8859(7) \\ \text { Cd2-I2 } & 2.7716(8) \\ \text { Cd2-I3 } & 2.7488(8) \\ \text { Cd2-I4 } & 2.7733(7) \\ & \\ \text { I1-Cd1-O1 } & 89.76(10) \\ \text { I1-Cd1-O3 } & 91.41(11) \\ \text { I1-Cd1-O5 } & 168.09(13) \\ \text { I1-Cd1-N1 } & 87.66(14) \\ \text { I1-Cd1-N2 } & 109.24(15) \\ \text { I1-Cd1-N3 } & 86.81(14) \\ \text { O1-Cd1-O3 } & 133.14(14) \\ \text { O1-Cd1-O5 } & 81.67(17) \\ \text { O1-Cd1-N1 } & 66.48(16) \\ \text { O1-Cd1-N2 } & 151.51(15) \\ \text { O1-Cd1-N3 } & 69.85(17) \\ \text { O3-Cd1-O5 } & 88.49(17) \\ \text { O3-Cd1-N1 } & 66.77(17) \\ \text { O3-Cd1-N2 } & 69.28(15) \\ \text { O3-Cd1-N3 } & 156.97(19) \\ \text { O5-Cd1-N1 } & 81.36(18) \\ \text { O5-Cd-N2 } & 81.8(2) \\ \text { O5-Cd-N3 } & 97.87(18) \\ \text { N1-Cd1-N2 } & 133.06(18) \\ \text { N1-Cd1-N3 } & 135.97(19) \\ \text { N2-Cd1-N3 } & 89.64(18) \\ \text { I1-Cd2-I2 } & 106.63(3) \\ \text { I1-Cd2-I3 } & 111.67(2) \\ \text { I1-Cd2-14 } & 103.64(2)\end{array}$


I2-Cd2-13

$12-\mathrm{Cd} 2-14$

$13-\mathrm{Cd} 2-14$

Cd1-I1-Cd2
$115.48(3)$
$108.26(2)$
$110.41(3)$
$113.73(2)$

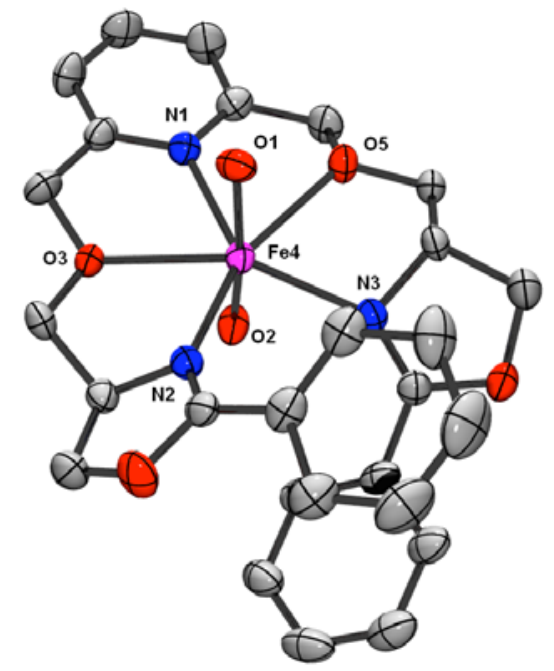

ORTEP-plot of $\left[\mathrm{Fe}(\mathbf{1})\left(\mathrm{H}_{2} \mathrm{O}\right)_{2}\right]^{2+}$ (3) (50\% probability level, hydrogens omitted for clarity) with atom numbering scheme.
$\mathrm{Fe}(4)-\mathrm{O}(1)$
2.111(3)
$\mathrm{Fe}(4)-\mathrm{O}(2)$
$2.148(3)$
$\mathrm{Fe}(4)-\mathrm{O}(3)$
2.332(3)
$\mathrm{Fe}(4)-\mathrm{O}(5)$
2.336(3)
$\mathrm{Fe}(4)-\mathrm{N}(1)$
2.339(3)
$\mathrm{Fe}(4)-\mathrm{N}(2)$
2.268(3)
$\mathrm{Fe}(4)-\mathrm{N}(3)$
2.232(3)
$\mathrm{O}(1)-\mathrm{Fe}(4)-\mathrm{O}(2)$
168.99(14)
$\mathrm{O}(1)-\mathrm{Fe}(4)-\mathrm{O}(3)$
91.46(10)
$\mathrm{O}(1)-\mathrm{Fe}(4)-\mathrm{O}(5)$
$82.45(10)$
$\mathrm{O}(1)-\mathrm{Fe}(4)-\mathrm{N}(1)$
86.02(11)
$\mathrm{O}(1)-\mathrm{Fe}(4)-\mathrm{N}(2)$
$87.57(12)$
$\mathrm{O}(1)-\mathrm{Fe}(4)-\mathrm{N}(3)$
98.82(14)
$\mathrm{O}(2)-\mathrm{Fe}(4)-\mathrm{O}(3)$
83.94(12)
$\mathrm{O}(2)-\mathrm{Fe}(4)-\mathrm{O}(5)$
93.41(12)
$\mathrm{O}(2)-\mathrm{Fe}(4)-\mathrm{N}(1)$
82.98(14)
$\mathrm{O}(2)-\mathrm{Fe}(4)-\mathrm{N}(2)$
100.26(14)
$\mathrm{O}(2)-\mathrm{Fe}(4)-\mathrm{N}(3)$
89.48(15)
$\mathrm{O}(3)-\mathrm{Fe}(4)-\mathrm{O}(5)$
133.29(10)
$\mathrm{O}(3)-\mathrm{Fe}(4)-\mathrm{N}(1)$
66.92(10)
$\mathrm{O}(3)-\mathrm{Fe}(4)-\mathrm{N}(2)$
70.68(10)
$\mathrm{O}(3)-\mathrm{Fe}(4)-\mathrm{N}(3)$
154.32(10)
$\mathrm{O}(5)-\mathrm{Fe}(4)-\mathrm{N}(1)$
$66.47(10)$
$\mathrm{O}(5)-\mathrm{Fe}(4)-\mathrm{N}(2) \quad 153.96(10)$
$\mathrm{O}(5)-\mathrm{Fe}(4)-\mathrm{N}(3) \quad 71.75(10)$
$\mathrm{N}(1)-\mathrm{Fe}(4)-\mathrm{N}(2) \quad 136.89(10)$
$\mathrm{N}(1)-\mathrm{Fe}(4)-\mathrm{N}(3) \quad 136.89(10)$
$\mathrm{N}(2)-\mathrm{Fe}(4)-\mathrm{N}(3) \quad 86.22(10)$ 


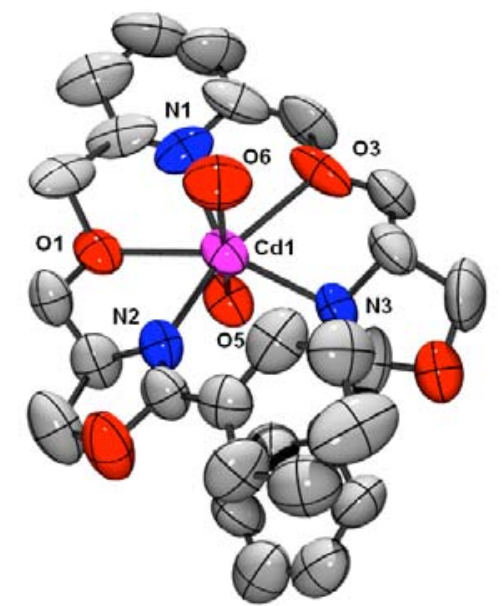

ORTEP-plot of $\left[\mathrm{Cd}(\mathbf{1})\left(\mathrm{H}_{2} \mathrm{O}\right)_{2}\right]^{2+}$

(4) (50\% probability level, hydrogens omitted for clarity) with atom numbering scheme.

$\begin{array}{ll}\mathrm{Cd}(1)-\mathrm{O}(6) & 2.325(11) \\ \mathrm{Cd}(1)-\mathrm{O}(5) & 2.303(9) \\ \mathrm{Cd}(1)-\mathrm{O}(1) & 2.455(10) \\ \mathrm{Cd}(1)-\mathrm{O}(3) & 2.413(10) \\ \mathrm{Cd}(1)-\mathrm{N}(1) & 2.356(15) \\ \mathrm{Cd}(1)-\mathrm{N}(2) & 2.350(15) \\ \mathrm{Cd}(1)-\mathrm{N}(3) & 2.348(12) \\ & \\ \mathrm{O}(6)-\mathrm{Cd}(1)-\mathrm{O}(5) & 170.9(5) \\ \mathrm{O}(6)-\mathrm{Cd}(1)-\mathrm{O}(1) & 98.3(4) \\ \mathrm{O}(6)-\mathrm{Cd}(1)-\mathrm{O}(3) & 81.6(6) \\ \mathrm{O}(6)-\mathrm{Cd}(1)-\mathrm{N}(1) & 84.9(5) \\ \mathrm{O}(6)-\mathrm{Cd}(1)-\mathrm{N}(2) & 87.0(6) \\ \mathrm{O}(6)-\mathrm{Cd}(1)-\mathrm{N}(3) & 97.4(4) \\ \mathrm{O}(5)-\mathrm{Cd}(1)-\mathrm{O}(1) & 79.4(3) \\ \mathrm{O}(5)-\mathrm{Cd}(1)-\mathrm{O}(3) & 93.6(5) \\ \mathrm{O}(5)-\mathrm{Cd}(1)-\mathrm{N}(1) & 86.2(4) \\ \mathrm{O}(5)-\mathrm{Cd}(1)-\mathrm{N}(2) & 100.2(5) \\ \mathrm{O}(5)-\mathrm{Cd}(1)-\mathrm{N}(3) & 88.0(4) \\ \mathrm{O}(1)-\mathrm{Cd}(1)-\mathrm{O}(3) & 132.9(5) \\ \mathrm{O}(1)-\mathrm{Cd}(1)-\mathrm{N}(1) & 64.3(4) \\ \mathrm{O}(1)-\mathrm{Cd}(1)-\mathrm{N}(2) & 69.4(4) \\ \mathrm{O}(1)-\mathrm{Cd}(1)-\mathrm{N}(3) & 154.4(3) \\ \mathrm{O}(3)-\mathrm{Cd}(1)-\mathrm{N}(1) & 68.7(6) \\ \mathrm{O}(3)-\mathrm{Cd}(1)-\mathrm{N}(2) & 156.2(5) \\ \mathrm{O}(3)-\mathrm{Cd}(1)-\mathrm{N}(3) & 69.6(5) \\ \mathrm{N}(1)-\mathrm{Cd}(1)-\mathrm{N}(2) & 131.1(5) \\ \mathrm{N}(1)-\mathrm{Cd}(1)-\mathrm{N}(3) & 137.4(4) \\ \mathrm{N}(2)-\mathrm{Cd}(1)-\mathrm{N}(3) & 91.4(4)\end{array}$




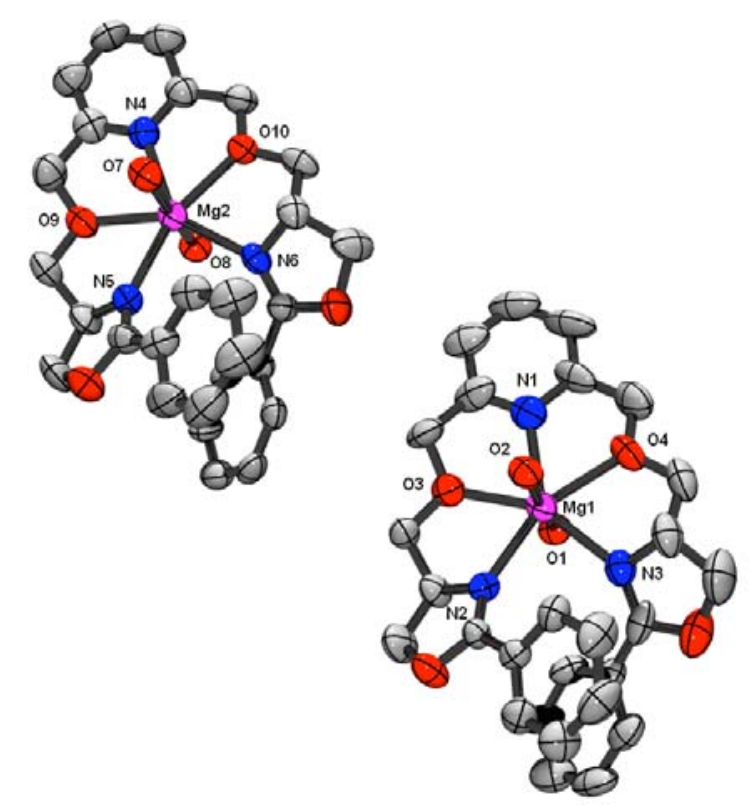

ORTEP-plot of the two unique units $\left[\mathrm{Mg}(\mathbf{1})\left(\mathrm{H}_{2} \mathrm{O}\right)_{2}\right]^{2+}(\mathbf{5})(50 \%$ probability level, hydrogens omitted for clarity) with atom numbering scheme.

$\begin{array}{llll}\mathrm{Mg}(1)-\mathrm{O}(1) & 2.062(5) & \mathrm{Mg}(2)-\mathrm{O}(7) & 2.054(5) \\ \mathrm{Mg}(1)-\mathrm{O}(2) & 2.055(4) & \mathrm{Mg}(2)-\mathrm{O}(8) & 2.072(4) \\ \mathrm{Mg}(1)-\mathrm{O}(4) & 2.292(5) & \mathrm{Mg}(2)-\mathrm{O}(9) & 2.261(5) \\ \mathrm{Mg}(1)-\mathrm{O}(3) & 2.274(5) & \mathrm{Mg}(2)-\mathrm{N}(4) & 2.239(5) \\ \mathrm{Mg}(1)-\mathrm{N}(1) & 2.326(6) & \mathrm{Mg}(2)-\mathrm{N}(5) & 2.327(6) \\ \mathrm{Mg}(1)-\mathrm{N}(2) & 2.310(7) & & 2.361(6) \\ \mathrm{Mg}(1)-\mathrm{N}(3) & 2.305(6) & \mathrm{O}(2)-\mathrm{N}(6) & 2.296(7) \\ & & \mathrm{O}(7)-\mathrm{Mg}(2)-\mathrm{O}(8) & 173.0(2) \\ \mathrm{O}(1)-\mathrm{Mg}(1)-\mathrm{O}(2) & 173.2(2) & \mathrm{O}(7)-\mathrm{Mg}(2)-\mathrm{O}(10) & 93.22(18) \\ \mathrm{O}(1)-\mathrm{Mg}(1)-\mathrm{O}(4) & 92.54(18) & \mathrm{O}(7)-\mathrm{Mg}(2)-\mathrm{N}(4) & 86.53(17) \\ \mathrm{O}(1)-\mathrm{Mg}(1)-\mathrm{O}(3) & 83.44(10) & \mathrm{O}(7)-\mathrm{Mg}(2)-\mathrm{N}(5) & 85.01(18) \\ \mathrm{O}(1)-\mathrm{Mg}(1)-\mathrm{N}(1) & 85.72(19) & \mathrm{O}(7)-\mathrm{Mg}(2)-\mathrm{N}(6) & 98.19(19) \\ \mathrm{O}(1)-\mathrm{Mg}(1)-\mathrm{N}(3) & 87.93(19) & \mathrm{O}(8)-\mathrm{Mg}(2)-\mathrm{O}(9) & 84.21(17) \\ \mathrm{O}(1)-\mathrm{Mg}(1)-\mathrm{N}(2) & 98.19(18) & \mathrm{O}(8)-\mathrm{Mg}(2)-\mathrm{O}(10) & 92.40(17) \\ \mathrm{O}(2)-\mathrm{Mg}(1)-\mathrm{O}(4) & 85.00(17) & \mathrm{O}(8)-\mathrm{Mg}(2)-\mathrm{N}(4) & 86.48(18) \\ \mathrm{O}(2)-\mathrm{Mg}(1)-\mathrm{O}(3) & 93.91(18) & \mathrm{O}(8)-\mathrm{Mg}(2)-\mathrm{N}(5) & 100.22(19) \\ \mathrm{O}(2)-\mathrm{Mg}(1)-\mathrm{N}(1) & 87.51(19) & \mathrm{O}(8)-\mathrm{Mg}(2)-\mathrm{N}(6) & 87.08(18) \\ \mathrm{O}(2)-\mathrm{Mg}(1)-\mathrm{N}(3) & 97.14(19) & \mathrm{O}(9)-\mathrm{Mg}(2)-\mathrm{O}(10) & 135.83(18) \\ \mathrm{O}(2)-\mathrm{Mg}(1)-\mathrm{N}(2) & 86.87(17) & \mathrm{O}(9)-\mathrm{Mg}(2)-\mathrm{N}(4) & 67.83(19) \\ \mathrm{O}(4)-\mathrm{Mg}(1)-\mathrm{O}(3) & 135.8(2) & \mathrm{O}(9)-\mathrm{Mg}(2)-\mathrm{N}(5) & 71.07(19) \\ \mathrm{O}(4)-\mathrm{Mg}(1)-\mathrm{N}(1) & 68.1(2) & \mathrm{O}(9)-\mathrm{Mg}(2)-\mathrm{N}(6) & 150.8(2) \\ \mathrm{O}(4)-\mathrm{Mg}(1)-\mathrm{N}(3) & 70.2(2) & \mathrm{O}(10)-\mathrm{Mg}(2)-\mathrm{N}(4) & 68.00(19) \\ \mathrm{O}(4)-\mathrm{Mg}(1)-\mathrm{N}(2) & 151.3(2) & \mathrm{O}(10)-\mathrm{Mg}(2)-\mathrm{N}(5) & 151.8(2) \\ \mathrm{O}(3)-\mathrm{Mg}(1)-\mathrm{N}(1) & 67.7(2) & \mathrm{O}(10)-\mathrm{Mg}(2)-\mathrm{N}(6) & 72.26(19) \\ \mathrm{O}(3)-\mathrm{Mg}(1)-\mathrm{N}(3) & 152.7(2) & \mathrm{N}(4)-\mathrm{Mg}(2)-\mathrm{N}(5) & 137.4(2) \\ \mathrm{O}(3)-\mathrm{Mg}(1)-\mathrm{N}(2) & 72.10(19) & \mathrm{N}(4)-\mathrm{Mg}(2)-\mathrm{N}(6) & 139.4(2) \\ \mathrm{N}(1)-\mathrm{Mg}(1)-\mathrm{N}(3) & 137.4(2) & \mathrm{N}(5)-\mathrm{Mg}(2)-\mathrm{N}(6) & 83.2(2) \\ \mathrm{N}(1)-\mathrm{Mg}(1)-\mathrm{N}(2) & 138.9(2) & & \\ \mathrm{N}(3)-\mathrm{Mg}(1)-\mathrm{N}(2) & 83.67(19) & & \\ & & & \end{array}$


2. X-ray crystallographic data collection and refinement details for $2 \mathrm{c}$ and $3-5$

\begin{tabular}{|c|c|c|c|c|}
\hline & $\begin{array}{c}\left.\mathrm{Fe}(\mathbf{1})\left(\mathrm{H}_{2} \mathrm{O}\right)_{2}\right]\left(\mathrm{ClO}_{4}\right)_{2} \\
{ }^{*} \mathrm{THF} \\
(\mathbf{3})\end{array}$ & $\begin{array}{c}\left.\left[\mathrm{Cd}(\mathbf{1})\left(\mathrm{H}_{2} \mathrm{O}\right)_{2}\right](\mathrm{ClO})_{4}\right)_{2} \\
{ }^{*} \times \text { solv. } \\
(\mathbf{4})\end{array}$ & $\begin{array}{c}\left.\mathrm{Mg}(\mathbf{1})\left(\mathrm{H}_{2} \mathrm{O}\right)_{2}\right]\left(\mathrm{ClO}_{4}\right)_{2} \\
{ }^{*} \times \text { solv. } \\
(\mathbf{5})\end{array}$ & $\begin{array}{c}{\left[\mathrm{Cd}(\mathbf{1})(\mu-\mathrm{l})\left(\mathrm{Cdl}_{3}\right)(\mathrm{MeOH})\right]} \\
{ }^{*} \mathrm{MeOH} \\
(\mathbf{2 c})\end{array}$ \\
\hline formula & $\mathrm{C}_{31} \mathrm{H}_{39} \mathrm{Cl}_{2} \mathrm{FeN}_{3} \mathrm{O}_{15}$ & $\begin{array}{c}\mathrm{C}_{27} \mathrm{H}_{31} \mathrm{CdCl}_{2} \mathrm{~N}_{3} \mathrm{O}_{14} \\
{ }^{*} \times \text { solv. }\end{array}$ & $\begin{array}{c}\mathrm{C}_{27} \mathrm{H}_{31} \mathrm{Cl}_{2} \mathrm{MgN}_{3} \mathrm{O}_{14} \\
\quad * \text { solv. }\end{array}$ & $\mathrm{C}_{29} \mathrm{H}_{35} \mathrm{Cd}_{2} \mathrm{I}_{4} \mathrm{~N}_{3} \mathrm{O}_{6}$ \\
\hline $\begin{array}{l}\text { crystal } \\
\text { appearance }\end{array}$ & colorless stick & colorless prism & colorless rod & colorless plate \\
\hline $\begin{array}{l}\text { mol. weight } \\
\text { crystal system }\end{array}$ & $\begin{array}{l}820.40 \\
\text { orthorhombic }\end{array}$ & $\begin{array}{l}804.86+x \text { solv } \\
\text { monoclinic }\end{array}$ & $\begin{array}{l}716.76+x . \text { solv. } \\
\text { monoclinic }\end{array}$ & $\begin{array}{l}1254.04 \\
\text { monoclinic }\end{array}$ \\
\hline space group & $\mathrm{P} 2{ }_{1} 2_{1} 2_{1}$ & $\mathrm{P} 2_{1}$ & $\mathrm{P} 2_{1}$ & $\mathrm{P} 2_{1}$ \\
\hline$a[\AA ̊]$ & $8.2616(7)$ & $8.6140(13)$ & $8.6129(8)$ & $12.1883(13)$ \\
\hline$b[\AA]$ & $14.0004(10)$ & $19.322(2)$ & $19.3622(11)$ & $13.5960(10)$ \\
\hline$c[\AA ̊]$ & $30.790(2)$ & $10.8199(19)$ & $20.792(2)$ & $12.9392(13)$ \\
\hline$\alpha\left[^{\circ}\right]$ & 90 & 90 & 90 & 90 \\
\hline$\beta\left[^{\circ}\right]$ & 90 & $107.088(19)$ & $96.846(11)$ & $115.881(11)$ \\
\hline$V\left[^{\circ}\right]$ & 90 & 90 & 90 & 90 \\
\hline volume $\left[\AA^{3}\right]$ & $3561.3(5)$ & $1721.4(5)$ & $3442.7(5)$ & 1929.1(4) \\
\hline z & 4 & 2 & 4 & 2 \\
\hline$\rho\left[\mathrm{g} \mathrm{cm}^{-3}\right]$ & 1.530 & 1.545 & 1.418 & 2.145 \\
\hline$\mu\left[\mathrm{mm}^{-1}\right]$ & 0.648 & 0.857 & 0.279 & 4.342 \\
\hline crystal size [mm] & $0.34^{\star} 0.08^{*} 0.06$ & $0.32^{*} 0.28^{*} 0.20$ & $0.16^{*} 0.06^{\star} 0.04$ & $0.24^{\star} 0.22^{\star} 0.06$ \\
\hline temperature $[\mathrm{K}]$ & $173(1)$ & $173(1)$ & $173(1)$ & $173(1)$ \\
\hline radiation $[\AA]]$ & $\operatorname{Mo}_{K \alpha}(\lambda=0.71073)$ & $\operatorname{Mo}_{K \alpha}(\lambda=0.71073)$ & $\operatorname{Mo}_{K \alpha}(\lambda=0.71073)$ & $\operatorname{Mo}_{K \alpha}(\lambda=0.71073)$ \\
\hline$\theta \max \left[{ }^{\circ}\right]$ & $1.97<\theta<25.81$ & $2.23<\theta<25.91$ & $2.32<\theta<25.30$ & $1.92<\theta<25.86$ \\
\hline $\begin{array}{l}\text { measured } \\
\text { reflections }\end{array}$ & 30644 & 15163 & 23013 & 13660 \\
\hline $\begin{array}{l}\text { independent } \\
\text { reflections }\end{array}$ & 6802 & 6566 & 11230 & 7085 \\
\hline $\begin{array}{l}\text { reflections } \\
\text { in refinement }\end{array}$ & $4743, I \geq 2 \sigma(I)$ & $4211, I \geq 2 \sigma(I)$ & $4892, I \geq 2 \sigma(I)$ & $6816, I \geq 2 \sigma(I)$ \\
\hline$R^{[a]}$ & 0.0443 & 0.0937 & 0.0605 & 0.0295 \\
\hline$w R_{2}^{[b]}$ & 0.0884 & 0.2477 & 0.1209 & 0.0764 \\
\hline$R^{[\mathrm{a}]}$ (all data) & 0.0723 & 0.1179 & 0.1197 & 0.0307 \\
\hline$w R_{2}$ (all data) & 0.0963 & 0.2658 & 0.1359 & 0.0770 \\
\hline $\begin{array}{l}\text { absolute } \\
\text { structure param. }\end{array}$ & $-0.01(2)$ & $0.13(7)$ & $0.16(8)$ & $0.00(2)$ \\
\hline $\begin{array}{l}\text { [a] } R \text { factor definiti } \\
\left.\mathrm{w}\left(\mathrm{F}_{0}{ }^{4}\right)\right] 1 / 2 \text {. Weight }\end{array}$ & $\left.R=\Sigma\left(\| \mathrm{F}_{0}|-| \mathrm{F}_{\mathrm{c}} \mid\right)\right)$ & $\left|F_{0}\right|$. [b] SHELX-97 & $\begin{array}{l}\text { factor definition: } u \\
\left.={ }_{c}^{2}\right] / 3 .\end{array}$ & $=\left[\Sigma \mathrm{w}\left(\mathrm{F}_{0}^{2}-\mathrm{F}_{\mathrm{c}}^{2}\right) 2 / \Sigma\right.$ \\
\hline
\end{tabular}


3. NMR spectra of compounds $(R, R)-1,2 \mathrm{c}$ and 4

Ligand $(R, R)-1$

${ }^{1} \mathrm{H}-\mathrm{NMR}\left(300 \mathrm{MHz}, \mathrm{CD}_{3} \mathrm{CN}\right)$

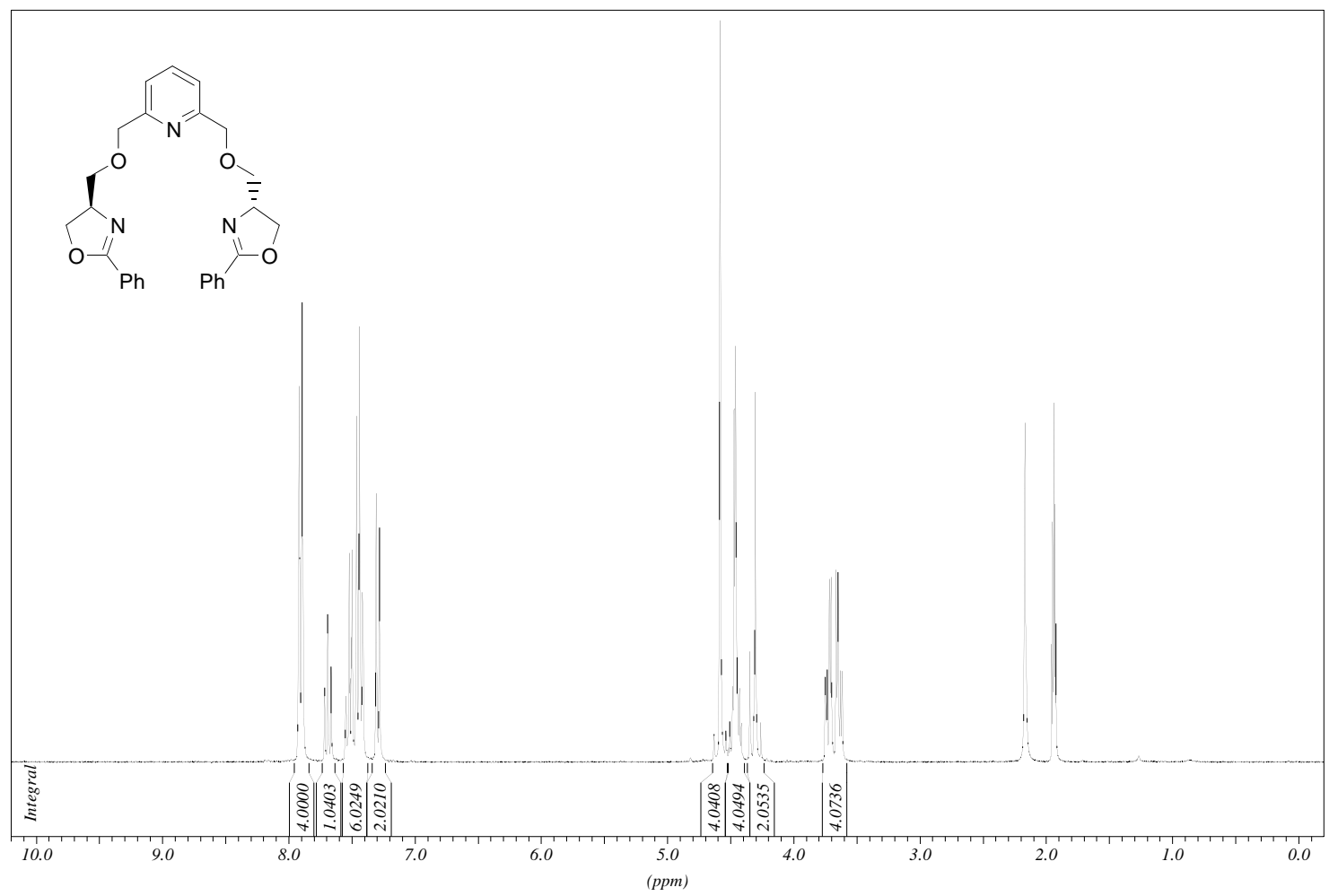

${ }^{13} \mathrm{C}-\mathrm{NMR}\left(75.5 \mathrm{MHz}, \mathrm{CD}_{3} \mathrm{CN}\right)$

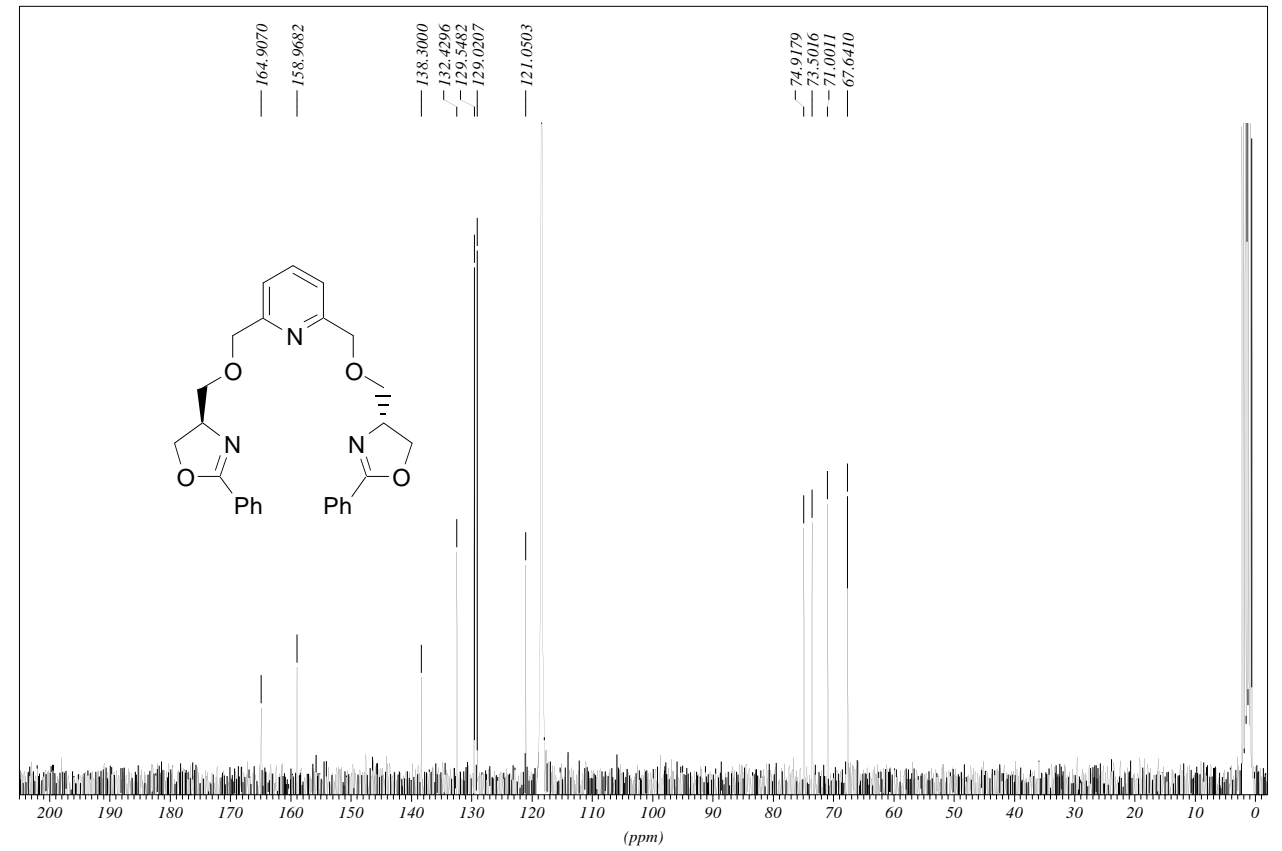


$\left[\mathrm{Cd}((R, R)-1)(\mathrm{MeOH})(\mu-\mathrm{I})\left(\mathrm{CdI}_{3}\right)\right]^{\star} \mathrm{MeOH}(2 \mathrm{c})$

${ }^{1} \mathrm{H}-\mathrm{NMR}\left(300 \mathrm{MHz}, \mathrm{CD}_{3} \mathrm{CN}\right)$

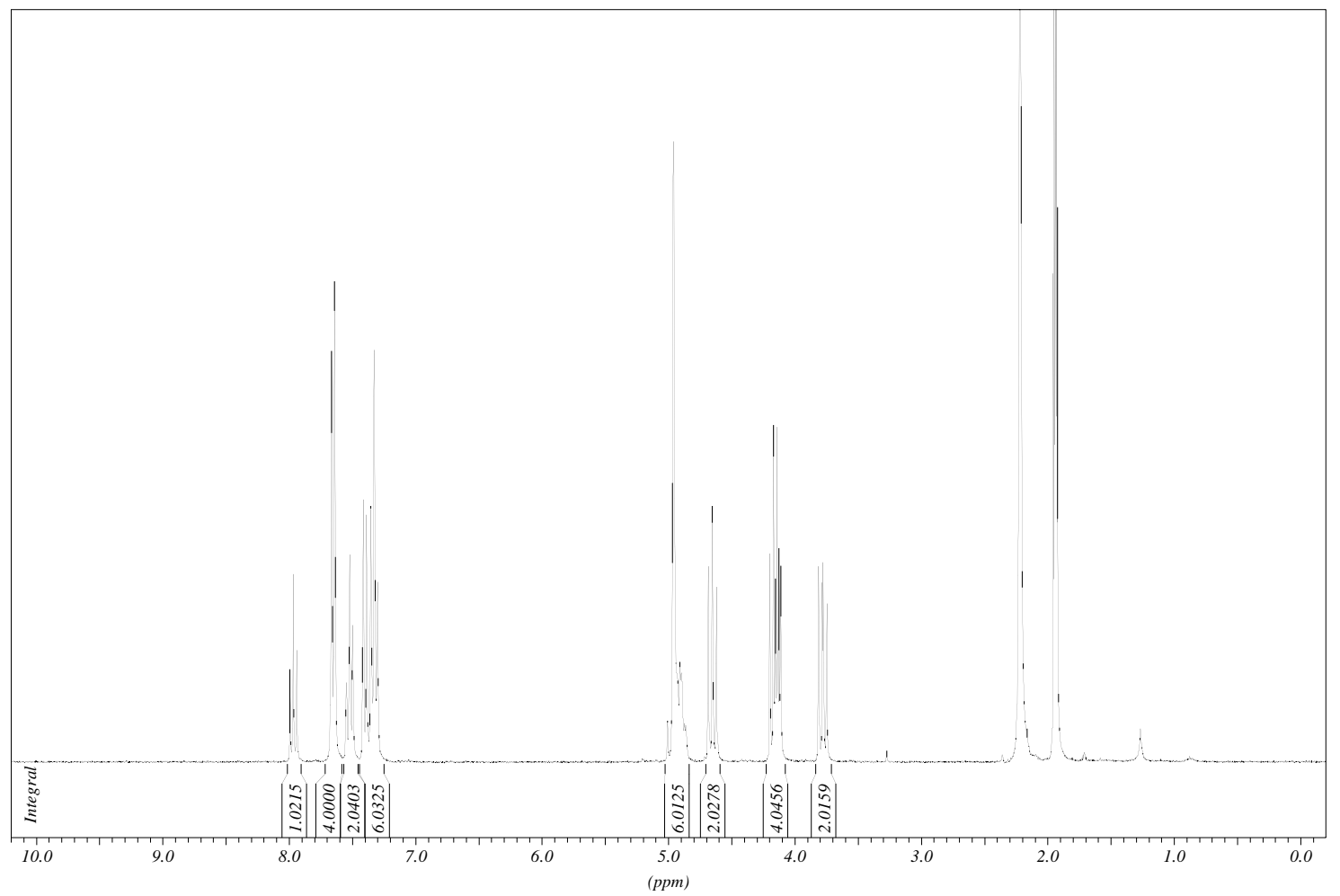

${ }^{13} \mathrm{C}-\mathrm{NMR}\left(75.5 \mathrm{MHz}, \mathrm{CD}_{3} \mathrm{CN}\right)$

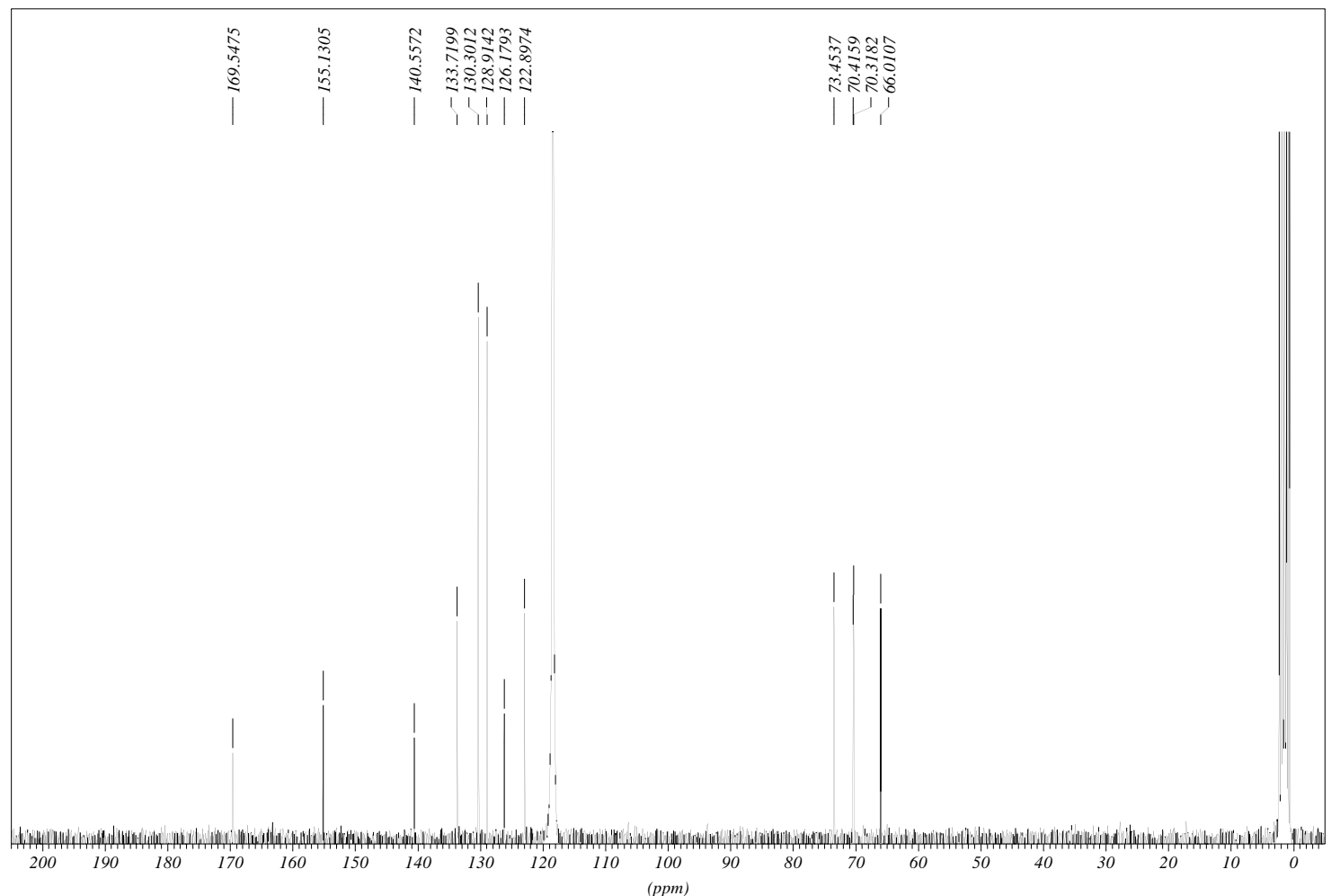


$\left[\mathrm{Cd}((\mathrm{R}, \mathrm{R})-1)\left(\mathrm{H}_{2} \mathrm{O}\right)_{2}\right]\left(\mathrm{ClO}_{4}\right)_{2}{ }^{*} \mathrm{x}$ solv. (4)

${ }^{1} \mathrm{H}-\mathrm{NMR}\left(300 \mathrm{MHz}, \mathrm{CD}_{3} \mathrm{CN}\right)$

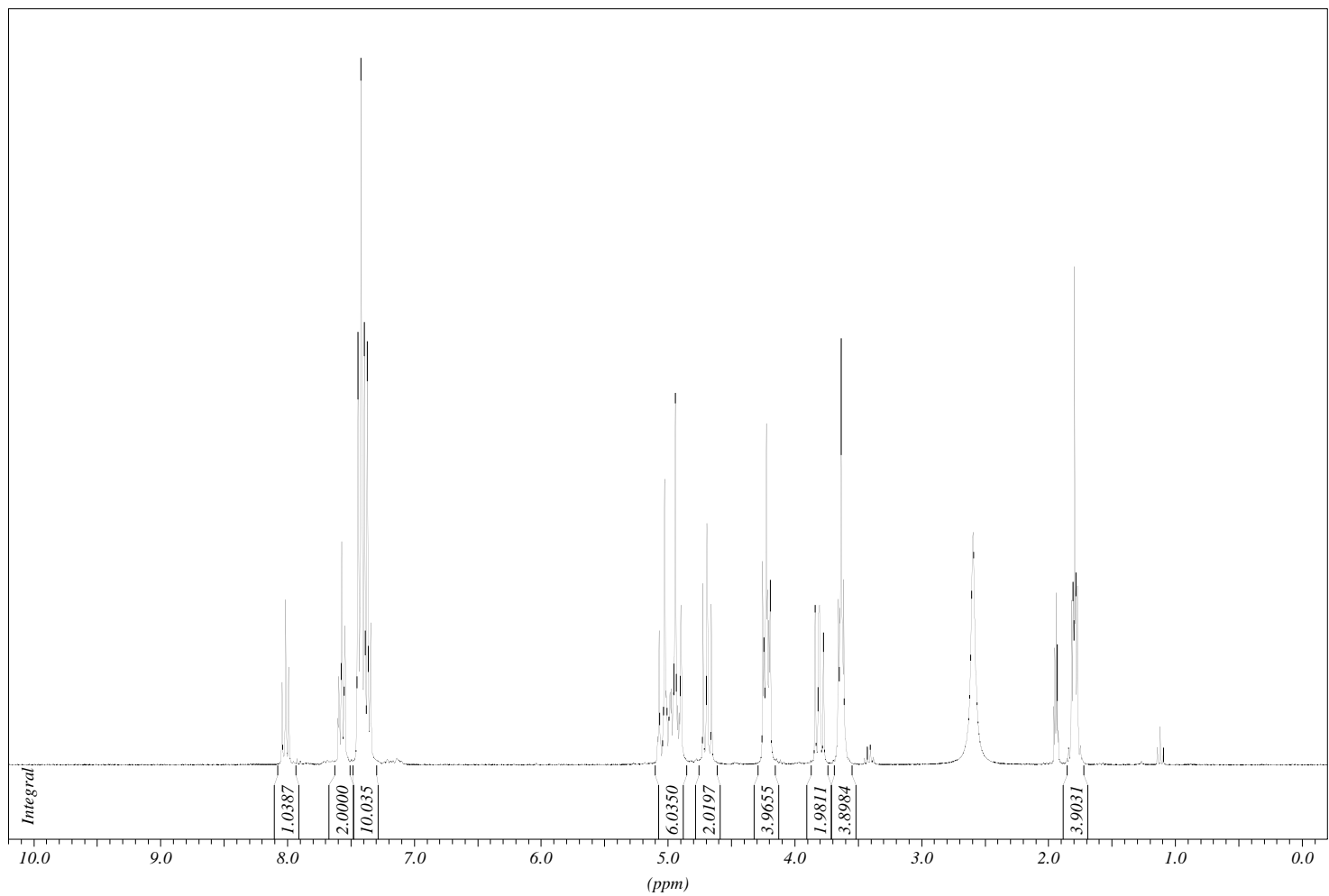

${ }^{13} \mathrm{C}-\mathrm{NMR}\left(75.5 \mathrm{MHz}, \mathrm{CD}_{3} \mathrm{CN}\right)$

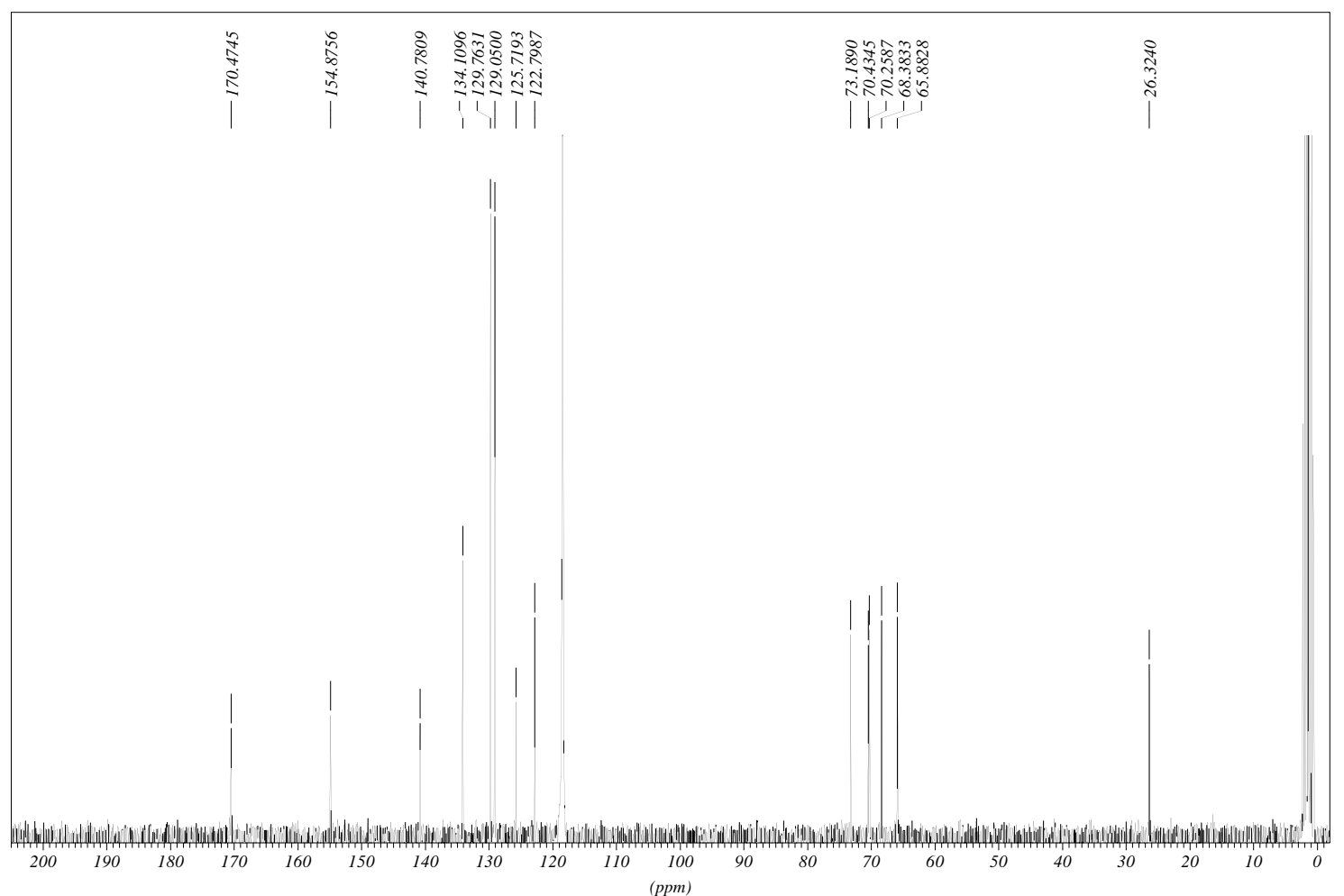

